\title{
Evaluation of HPV Molecular Tests in Primary Screening for Cervical Cancer in Brazil
}

\author{
José E. Levi1,2*, Adhemar Longatto-Filho1,3,4,5, José Eluf-Neto1,6, Célia L. Rodrigues, \\ Cristina M. Oliveira ${ }^{7}$, Adriana C. Carlonii ${ }^{1,5}$, Adriana T. Lorenzi ${ }^{1,5}$, Maricy Tacla ${ }^{1,8}$, \\ José H. Fregnani ${ }^{1,9}$, Alexandre M. Ab'Saber ${ }^{10}$, Cristovam Scapulatempo ${ }^{9}$, Luisa L. Villa1,11,12 \\ ${ }^{1}$ National Institute of Science and Technology for the Diseases Associated to Papillomaviruses (INCT-HPV), \\ São Paulo, Brazil \\ ${ }^{2}$ Virology Lab, Tropical Medicine Institute, Universidade de São Paulo, São Paulo, Brazil \\ ${ }^{3}$ Laboratory of Medical Investigation (LIM) 14, Faculdade de Medicina, Universidade de São Paulo, \\ Sao Paulo, Brazil \\ ${ }^{4}$ ICVS/3B's-PT Government Associate Laboratory, Life and Health Sciences Research Institute (ICVS), \\ School of Health Sciences, University of Minho, Braga, Portugal \\ ${ }^{5}$ Molecular Oncology Research Center, Pio XII Foundation, Barretos, Brazil \\ ${ }^{6}$ Department of Preventive Medicine, Faculdade de Medicina, Universidade de São Paulo, São Paulo, Brazil \\ ${ }^{7}$ Department of Infectious Diseases, Faculdade de Medicina, Universidade de São Paulo, São Paulo, Brazil \\ ${ }^{8}$ Department of Ginecology and Obstetrics, Faculdade de Medicina, Universidade de São Paulo, \\ Sao Paulo, Brazil \\ ${ }^{9}$ Center for the Researcher Support, Pio XII Foundation, Barretos Cancer Hospital, Barretos, Brazil \\ ${ }^{10}$ Department of Pathology, Faculdade de Medicina, Universidade de São Paulo, São Paulo, Brazil \\ ${ }^{11}$ Department of Radiology, Center on Translational Oncology Investigation, São Paulo State Cancer Institute, \\ Faculdade de Medicina, Universidade de São Paulo, São Paulo, Brazil \\ ${ }^{12}$ Santa Casa de São Paulo Medical School, São Paulo, Brazil \\ Email: ${ }^{*}$ dudilevi@usp.br
}

Received 11 March 2014; revised 10 April 2014; accepted 18 April 2014

Copyright (C) 2014 by authors and Scientific Research Publishing Inc.

This work is licensed under the Creative Commons Attribution International License (CC BY).

http://creativecommons.org/licenses/by/4.0/

cc) (7)

\section{Abstract}

Background: Incorporation of HPV tests into cervical cancer screening programs may be advantageous over conventional cytology, especially in developing nations, where the largest burden of cervical cancer is observed. Objectives: To conduct an evaluation of commercially available molecular HPV tests in Brazilian women. Study design: Two groups were recruited: group A was composed of 511 women referred to the clinics because of a previous abnormal Pap test while group B

\footnotetext{
*Corresponding author.
} 
consisted of 2464 subjects under routine screening. Cervical samples were collected using SurePath liquid cytology (LBC) device, and split into aliquots which were submitted to molecular testing by Hybrid Capture and cobas HPV. Colposcopy and biopsies were made according to the standard guidelines, directed by cytological diagnosis. Results: Prevalence of HSIL was $5.97 \%$ and $0.7 \%$ in Group A and B respectively. High-Risk HPV DNA was found in about $9 \%$ of group B women, while in group A this frequency was $\mathbf{2 4 \%}$. Having CIN3+ as the study end-point, the negative predictive values for molecular methods were above $99.8 \%$. All "in-situ" and invasive cervical carcinomas were detected by both HPV nucleic acid assays. Conclusion: Use of HPV DNA testing was feasible and highly sensitive in cancer screening settings of Brazil.

\section{Keywords}

\section{High-Risk HPV Test, HPV-DNA, Cervical Cancer Screening}

\section{Introduction}

Since the establishment of an etiological link between high-risk Human Papillomaviruses (HR-HPV) and cervical cancer [1] (CC), and its recognition by all players involved in control, treatment and research of this common neoplasia [2], the dominant role of cytological screening in cervical cancer prevention was challenged by molecular methods based on the detection of viral nucleic acids [3] (Nucleic Acid Testing).

As a parallel, screening of blood and organ donors by NAT for the Hepatitis C and Human Immunodeficiency viruses RNAs has been adopted worldwide years ago [4]. Differently from CC screening, NAT has not replaced conventional immunoassays but added, in order to cover the window-period, experienced by recently infected donors preceding seroconversion.

In certain settings, HPV testing has been used as an adjuvant to cytology, and many guidelines include and recommend such use [5]. However, replacement of routine cytological screening by HPV NATs has been a matter of passionate debates. Many large studies were carried in order to evaluate adequately this important public health issue. Most studies converged to a common conclusion in favor of primary screening by HPV NATs followed by different triage algorithms [6]-[9]. As HPV is prevalent among sexually active women, the specificity of HPV testing is clearly inferior to cytology. Nevertheless, the measurable gain in sensitivity surpasses the caveats of the inferior specificity. Moreover, mainly due to the possibility of safely extending the screening interval, HPV NATs are cost-effective in different scenarios [10]-[14]. For instance, the Netherlands was the first country to announce a complete shift towards CC screening by a polymerase chain reaction-enzyme immunoassay method (PCR + EIA), beginning in 2016 [15] [16]. Mexico introduced a successful program for women belonging to communities underserved by the health system, consisting of vaccinating the young, screening by Pap women in between 24 and 35 years old and screening by DNA testing those older [17].

Brazil launched a national CC screening program about 40 years ago. The reduction on CC mortality verified recently in a few state capitals may be partially attributed to this program, which has been intensified in the last decade [18]. Nevertheless, the incidence of CC in 2012 was estimated to be of about 17/100,000 women with huge regional differences. For instance, the estimated incidence for 2012 in Mato Grosso do Sul State was 39.4/100,000 while in São Paulo State it was 16.4/100,000 (age-unadjusted) [19].

In consonance to organized CC screening programs worldwide, Brazilian guideline recommends cytological testing every three years after two annual negative smears, for the targeted population of women in between 25 64 years old. About 11 million Pap smears were collected in 2009, suggesting the coverage of 66\% (50 million women corresponding to 16.6 million smears per year in a triennium period). However, in practice, there are many women that perform Pap smears yearly and also unscreened women, indicating that the population coverage shall be below $50 \%$ in some regions of the country. Of course, these unscreened women are the main source of new cases of CC and projects aiming to reach them and ensure their gynecological health is paramount. Some barriers to their access to the CC screening program may be overcome by the conjunction of self-collection devices and HPV DNA testing, as shown in other countries [20].

The aim of this study was to evaluate the performance of some of the commercially available HPV NATs for their sensitivity, specificity, predictive values, rate of invalid results and accuracy under routine CC screening 
conditions.

\section{Material and Methods}

\subsection{Ethics Statement}

This study was approved by the Ethical Committee of both participating institutions (Comitê de Ética em Pesquisa da Fundação Pio XII-Hospital de Câncer de Barretos, Project \#244/2009, approved in December $12^{\text {th }}$, 2009 and Comissão de Ética para Análise de Projetos de Pesquisa da Diretoria Clínica do Hospital das Clínicas e da Faculdade de Medicina da Universidade de São Paulo, CAPPesq, Project 24/10, approved in April $8^{\text {th }}$, 2010) and all patients enrolled provided written informed consent.

A subset of the 30,000 women enrolled in a liquid-cytology prospective multi-centre validationstudy(RODEO) [21], designed to evaluate the value of SurePath (Tripath Imaging Inc, Burlington, NC, USA), liquid-based cytology with FocalPoint (Tripath Imaging Inc, Burlington, NC, USA) was randomly assigned to the molecular testing protocol. Two centers participated in the study: Hospital das Clínicas from the São Paulo Medical School, University of São Paulo and Hospital do Câncer de Barretos, Barretos, São Paulo State.

Between October 2009 and April 2011 both centers recruited women from two groups. Group A was composed of 511 women with a previous abnormal Pap test in the year preceding study enrollment (354 from Barretos and 157 from São Paulo) while Group B consisted of 2464 patients (1964 from Barretos and 500 from São Paulo) undergoing routine screening.

Mean and median age was respectively 40.1 (sd \pm 12.9 years) and 47.0 years for the Group A and 42.7 (sd \pm 14.4 years) and 46.7 years for the Group B women. Cervical samples were collected using SurePath liquid cytology collection device, and processed according to the manufacturer protocol. The remaining cell pellet was ressuspended in Surepath preservative fluid, split into 4 aliquots and stored frozen at $-20^{\circ} \mathrm{C}$. Each sample was thawed and processed for the NAT according to manufacturer instructions. For the cobas 4800 it was used the cell suspension in SurePath liquid, whereas for Hybrid Capture,a protocol for sample processing was provided by Qiagen. Finally, all positive samples by either test were genotyped by the PapilloCheck kit (Greiner BioOne).

\subsection{Statistics}

Mean, median and standard deviations were calculated using Excel (Microsoft Co., USA) while confidence intervals of $95 \%$ were obtained by applying the method of Newcombe for proportions, as described [22].

\subsection{HPV Molecular Tests}

We chose to evaluate tests that were commercially available and based on different targets. Hybrid Capture employs RNA probes that hybridize all over the HPV genome, cobas 4800 uses L1 primers and probes specific for HPV 16 and 18 and another 12 HPV types in addition to a cellular control (beta-globin).

Hybrid Capture 2 (Qiagen, Gaithersburg, MD, USA) — This is a nucleic acid hybridization assay with signal amplification that utilizes microplate chemiluminescent detection. Specimens containing target DNA hybridize with a specific HPV RNA probe cocktail further detected by an antibody against RNA:DNA hybrids. This test detects HR-HPV genotypes 16, 18, 31, 33, 35, 39, 45, 51, 52, 56, 58, 59 and 68.

Cobas 4800 HPV Test (Roche Molecular Systems, Branchburg, NJ, USA) is a Real-Time PCR assay that identifies HPV types by using type-specific probes for HPVs 16 and 18, plus a group of 12 HR-HPV types 31, 33, 35, 39, 45, 51, 52, 56, 58, 59, 66 and 68 (High-Risk HR probe) in addition to beta-globin as a specimen cellular content control.

\subsection{Cytology and Histology}

Smears were examined by regular cytologists at both institutions and classified according to the Bethesda system. Altered smears were reviewed by one of the authors (AL). When discrepancies occurred, the assessment made by AL prevailed. Colposcopy and biopsies were made according to the standard guidelines, guided by cytological results; cytological high grade lesions (Figure 1) were submitted to colposcopic evaluation and biopsies taken when suggestive lesions visualized.HPV testing results were unavailable to clinicians and did not influence patient's management. 


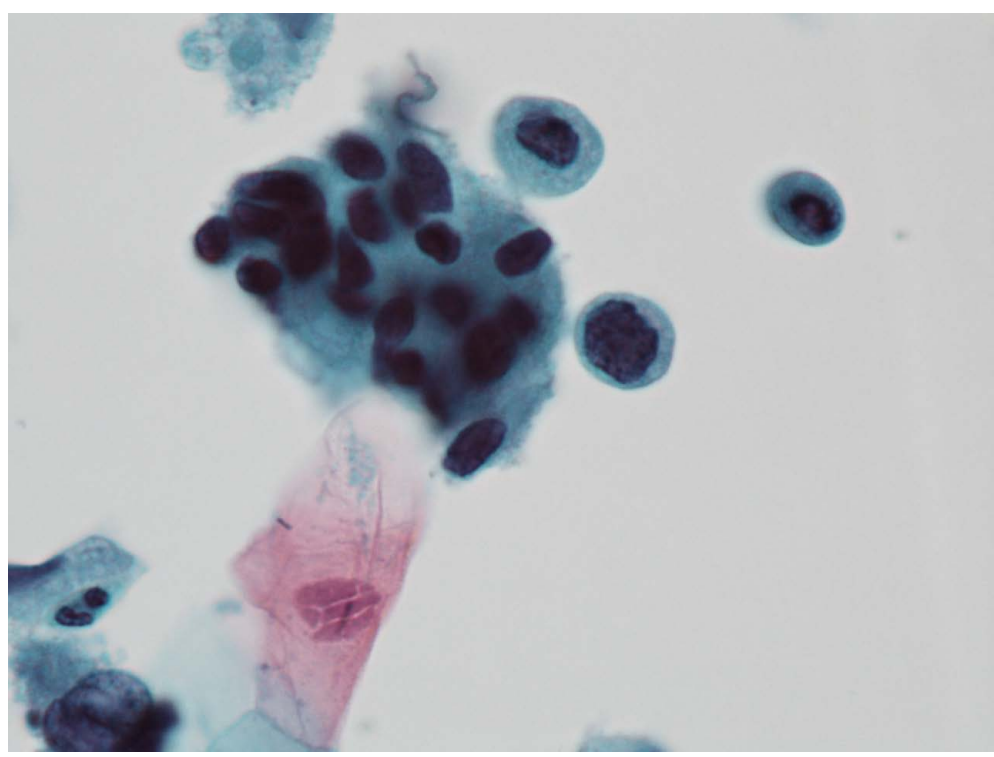

Figure 1. High grade lesion from Surepath (BD, Burlington, USA) liquidbase preparation showing rounded cells with single dyskariotic nuclei with irregular borders, surrounded by a group of dyskariotic nuclei. Papanicolaou stain, original magnification $\times 400$.

\section{Results}

\subsection{Cytology and Histology}

Group B patients followed the common distribution of cytological results on screening populations whereas Group A displayed a much higher prevalence of cytological abnormalities, including cervical carcinomas, as shown in Table 1. Overall, 28 women had an altered smear in Group B and 123 in Group A (Table 2). These patients were invited to a second visit when colposcopy was performed and biopsies taken, if indicated. Among Group A subjects, one-hundred and eleven women attended to this visit $(111 / 123=90 \%)$. In Group B, 81 women had smears > negative for intraepithelial lesion or malignancy (NILM) but only 28 patients were biopsied $(28 / 81=34.5 \%)$. This difference is mainly due to the policy in Barretos of not referring to colposcopy patients with low-grade squamous intraepithelial lesion (LSIL) or atypical squamous cells of undetermined significance (ASCUS) smears but also to patients that failed to return.

Concordance between cytology and histology is hampered by the significant number of high-grade squamous intraepithelial lesion (HSIL) samples not submitted to colposcopy/biopsy (10/18 HSILs). The gold-standard against which molecular tests were evaluated is histology confirmed cervical intraepithelial neoplasia (CIN) 2/3+. Overall, there were 18 cases of CIN2, 24 CIN3 and 7 invasive carcinomas, including 1 adenocarcinoma. Among the 31 CIN3+ cases (Figure 2), four came from the screening population, two CIN3, one adenocarcinoma and one squamous cell carcinoma. One endometrial carcinoma and one adenosarcoma were detected as well but were not considered for the purpose of this evaluation.

\subsection{Molecular Tests}

Figure 3 shows the HR-HPV prevalence verified by each method on both study groups. The prevalence of HRHPVs in the screening population, as depicted by the cobas 4800 was $7.6 \%$ (CI 95\% 6.6\% - 8.7\%) HPV $161.9 \%$ (CI 95\% 1.4\% - 2.5\%) and HPV 18 0.8\% (CI 95\% 0; 49\% - 1.2\%). The sum exceeds 9.7\% (total HPV prevalence by cobas 4800 in Figure 3) due to co-infections.

Age distribution of HR-HPV DNA frequency on the screening group (B) follows that commonly observed in the general population, i.e. higher rates in younger women, decreasing significantly over 30 years old (Figure 4). A peak of $43 \%$ can be seen on women in between 61 - 65 years old in the referral population (group A), probably a chance event caused by the small sample size of this age category $(\mathrm{N}=21)$.

Sensitivity of Hybrid Capture and cobas $4800 \mathrm{HPV}$ was identical; both failed to detect 5 CIN3+ cases out of 


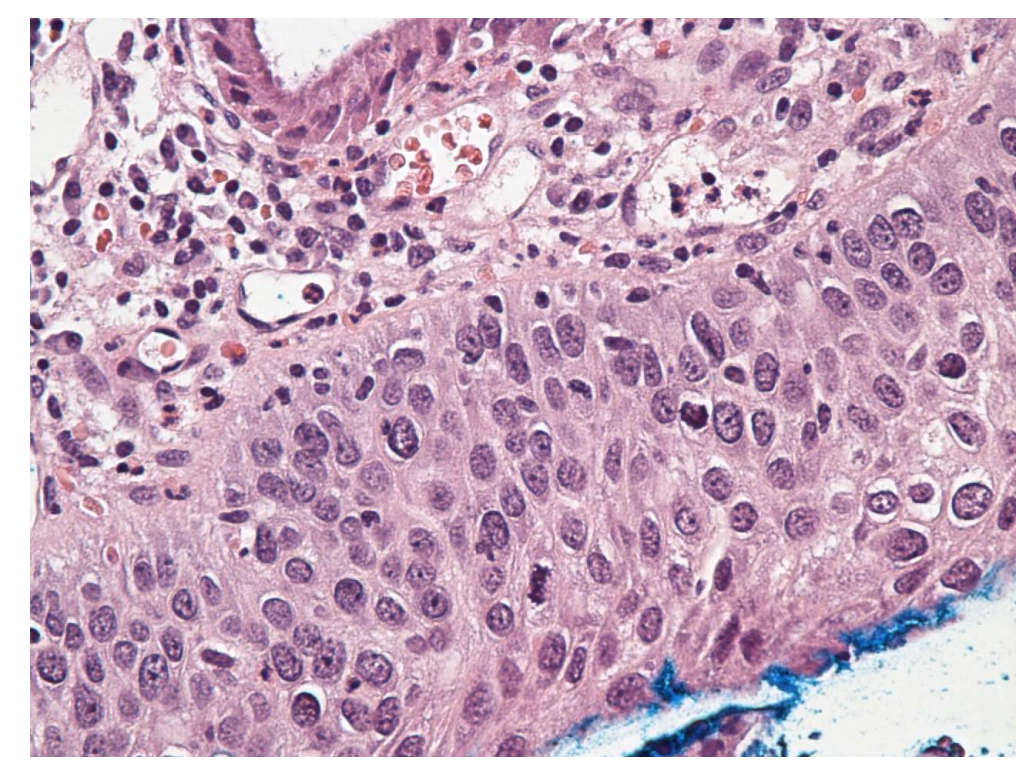

Figure 2. Severe cervical intraepithelial lesion exhibiting extensive cellular alterations and atypical mitoses. H\&E Stain, original magnification $\times 100$.

Table 1. Frequency of cytological categories according to the Bethesda system in Group A (referral) and Group B (screening) cohorts. NILM = Negative for Intraepithelial Lesion or Malignancy; LSIL = Low-grade Squamous Intra-epithelial Lesion; ASCUS = Atypical Squamous Cells of Undetermined Significance; HSIL = High-Grade Squamous Intra-Epithelial Lesion; ICC = Invasive Cervical Carcinoma; NR = No Result.

\begin{tabular}{ccccc}
\hline & CIN 1 & CIN 2 & CIN 3 & ICC \\
\hline GROUP B $(\mathrm{N}=28)$ & $0.06 \%$ & $0.0 \%$ & $0.1 \%$ & $0.08 \%$ \\
GROUP A $(\mathrm{N}=112)$ & $9.0 \%$ & $3.5 \%$ & $4.3 \%$ & $1.0 \%$ \\
\hline
\end{tabular}

Table 2. Frequency of histological categories according to the WHO classification in Group A (referral) and Group B (screening) cohorts. CIN = Cervical Intraepithelial Neoplasia; ICC = Invasive Cervical Carcinoma.

\begin{tabular}{cccccccccc}
\hline & NILM & ASCUS & ASC-H & AGUS & LSIL & HSIL & ICC & NR & TOTAL \\
\hline $\begin{array}{c}\text { GROUP B } \\
(\mathrm{N}=\text { 2464) }\end{array}$ & $96.6 \%$ & $0.9 \%$ & $0.5 \%$ & $0.0 \%$ & $1.3 \%$ & $0.7 \%$ & $0.0 \%$ & $0.3 \%$ & $100 \%$ \\
$\begin{array}{c}\text { GROUP A } \\
(\mathrm{N}=511)\end{array}$ & $74.2 \%$ & $6.7 \%$ & $2.2 \%$ & $0.0 \%$ & $8.8 \%$ & $5.9 \%$ & $0.6 \%$ & $1.8 \%$ & $100 \%$ \\
\hline
\end{tabular}

31, though these 5 negatives were not coincidental. All carcinomas ("in situ" and invasive) were detected by the cobas 4800 and HC. Both methods presented negative predictive values of $99.8 \%$ as seen on Table 3.

HPV Type Distribution-All samples HR-HPV positive for at least one of the methods $(\mathrm{n}=328 \mathrm{CH}+; 350$ cobas $4800+$ ) plus a random subsample of 300 samples negative for both methods were submitted to HPV genotyping by the PapilloCheck test. Results are displayed on Figure 5. The presence of low-risk HPV genotypes in this figure is explained by the inclusion of probes for these genotypes in the PapilloCheck assay, depicting them when present in co-infections with the HR-HPV genotypes.

\section{Discussion}

Scientific evidence gathered in the last five years strongly advocates the use of HPV NAT as a tool for primary screening for cervical cancer [23]. Studies showed NAT to be more sensitive than standard cytology [24], detecting about $50 \%$ additional cases of CIN3+. In several countries, this gain in sensitivity was not accompanied by a substantial increase in referrals to colposcopy due to an HR-HPV positive result [25]. Longitudinal studies also evidenced that an screening interval of 5 - 6 years after an HR-HPV negative result was safe [8] [16], thus 


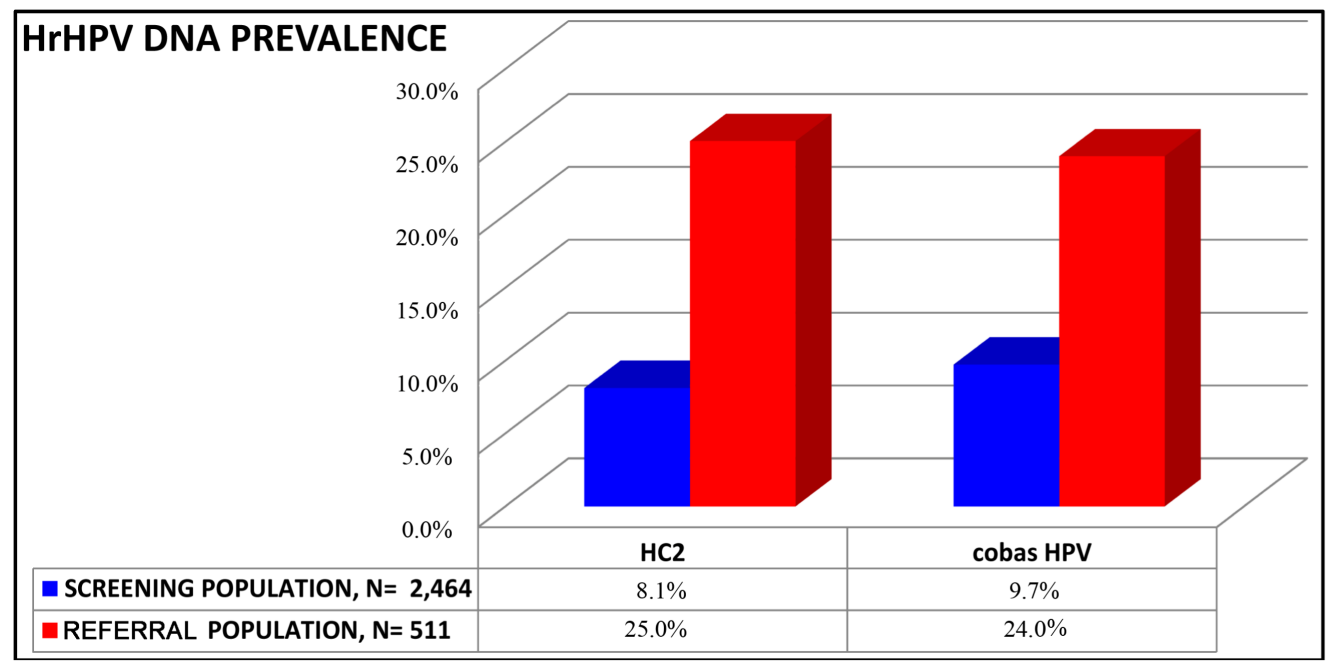

Figure 3. Prevalence of high-risk HPVs on Group A (referral) and Group B (screening) populations by method.

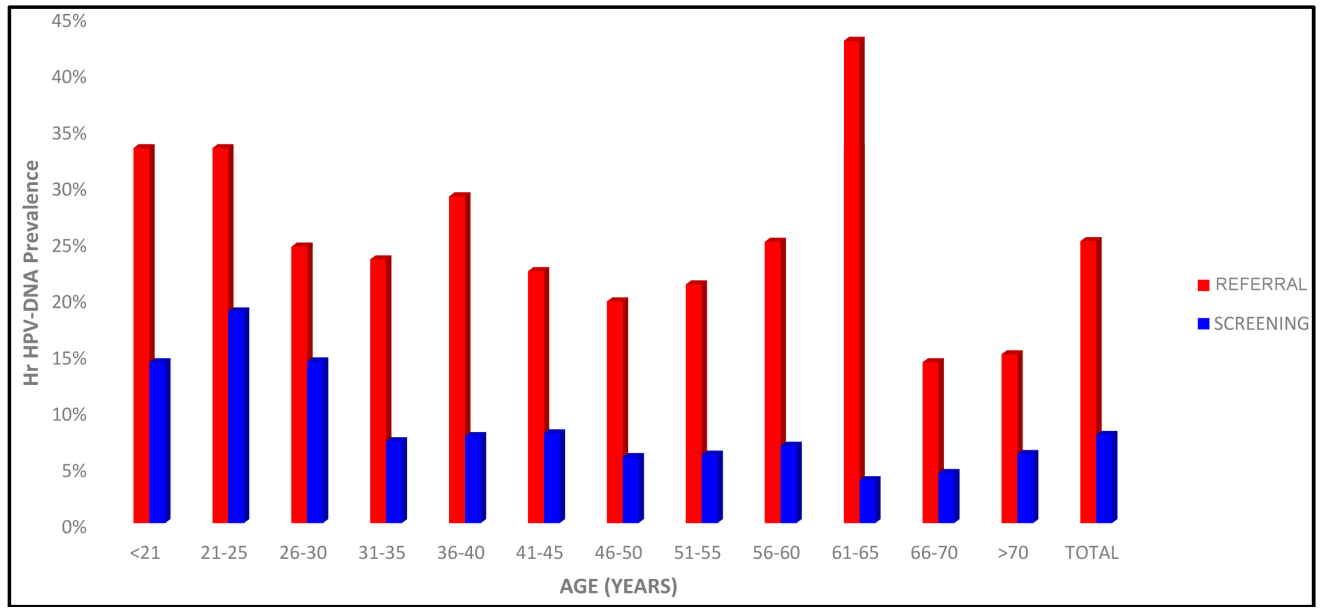

Figure 4. Age-distribution of HR-HPV prevalence on Group A (referral) and Group B (screening), as measured by the Hybrid Capture assay.

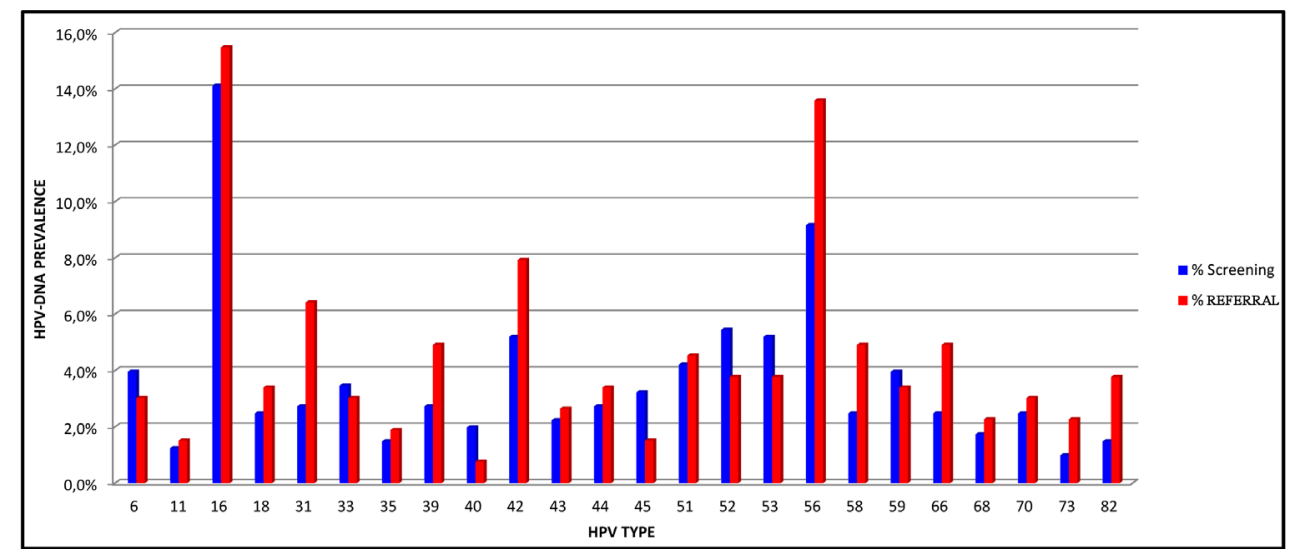

Figure 5. HPV Genotypes (determined by the PapilloCheck test) frequency distribution among subjects HR-HPV positive by one or more NAT screening assays in group A (referral) and group B (screening) populations. 
Table 3. Sensitivity, specificity, positive predictive value (PPV) and negative predictive value (NPV) for histology-proven CIN3+ for all molecular tests.

\begin{tabular}{ccccc}
\hline METHOD & SENSITIVITY & SPECIFICITY & POSITIVE PREDICTIVE & \multicolumn{2}{c}{ NEGATIVE PREDICTIVE } \\
VALUE & $\%(\mathrm{CI} 95 \%)$ & $\%(\mathrm{CI} 95 \%)$ \\
HC & $83.9(66.3-94.6)$ & $89.7(88.5-90.8)$ & $7.9(5.2-11.4)$ & $99.8(99.6-99.9)$ \\
COBAS & $83.9(66.3-94.6)$ & $88.7(87.4-89.8)$ & $7.5(5.0-10.9)$ & $99.8(99.5-99.9)$ \\
\hline
\end{tabular}

reducing the number of referrals and the overall cost of the screening programs.

Several HPV NATs were available in the market, and we evaluated methodologies with different targets and principles in a large number of patients. By including two groups with distinct epidemiological backgrounds, we aimed to assess specificity in the regular screening population and sensitivity in a population referred for a previous abnormal Pap test, as this would likely harbor a larger number of high-grade lesions. However, a caveat of such "split sample" study was the necessity to manipulate the samples, losing $100 \%$ traceability and potentially introducing human errors. Moreover, as we decided to use samples from another liquid based cytology study evaluating the SurePath system (BD, USA), our biological material was a pellet of epithelial cells in SurePath liquid preservative. This is not the material of choice for the NATs employed, as they all foresee the use of primary tubes, i.e. before any processing. Hybrid Capture 2 and cobas 4800 HPV test showed very similar performances (sensitivity and specificity for CIN3+ of $84 \%$ and $89 \%$ for both tests respectively) corroborating findings from other comparative evaluations [26]. Cobas 4800 offers the advantage of genotyping HPV 16 and 18 in the screening test and also contains a control for sample adequacy (beta-globin), being a fully automated platform. Reassuringly, HC2 and cobas 4800 detected all "in-situ" $(n=7)$ and invasive carcinomas $(n=7)$. Moreover, the negative predictive value was $99.8 \%$ for both methods, fulfilling one of the suggested requirements for their use as primary screening tools [27]. The HR-HPV prevalence in this population (group B, representing general screening population) of $8 \%-10 \%$ is similar to other Brazilian studies [28] [29]. Noticeably, HPV 56 appeared as the second more frequent type in both groups. Other studies using PapilloCheck for genotyping are also reporting rates of HPV 56 above the expectancy [30], what may be attributable to an increased sensitivity or reduced specificity of this test for this particular type. Being HR-HPV a common infection, the positive predictive value for CIN3+ is low, less than $10 \%$, meaning that only one in ten screen-positive women will have an HSIL detected on triage. It has been proposed that the judicious use of the genotyping and patient age information may increase the PPV, by directly referring to colposcopy only those above 30 years old and HPV 16 and/or 18 positive. Women with an HR-HPV result (non-16, non-18) would repeat HPV testing and concomitant cytology one year after [31]. Other countries simply decided to restrict test to women 30 years old and above. We believe this alternative is not applicable to the current situation in Brazil where national guidelines recommend screening to begin at the age of 25 years, although this service is not denied to younger women willing to be screened. Moreover, there is a small percentage (approx. 4\%., Levi et al. unpublished) of invasive cancers occurring on young women, below 30 years old, whom, in addition to those bearing high-grade precursor lesions, will certainly benefit from NAT screening. Accordingly, in this study, 5 out of the 31 CIN3+ patients were less than 30 years old, including two cases of carcinoma "in situ". In São Paulo state, adenocarcinomas are becoming proportionally more prevalent, and it is well recognized that the cytology is particularly less sensitive for the diagnosis of this histological type [8], whereas HPV NATs are supposed to show the same sensitivity for squamous and adenocarcinomas.

The major strengths of this study are the large number of patients enrolled and the significant number of cytological abnormalities verified, allowing the accurate assessment of the performance of NAT methods. However, several limitations must be acknowledged. First, we don't have any additional information from the women testing HR-HPV positive but negative by LBC, hindering a more comprehensive evaluation of the role of HPV testing in screening, in this population. Furthermore, the suboptimal biological specimen used may have contributed to some HPV NATs false-negatives observed. Finally, histology was adopted as the gold-standard for cervical disease, whereas two cases classified as CIN3+ were HPV negative by all methods. These could be, in fact, mistakes in the histopathological analysis instead of false-negative HPV-NATs.

We consider that São Paulo state may pioneer a shift in screening methods that will hopefully lead to a substantial reduction in the incidence and mortality from cervical cancer in Brazil, ultimately a preventable disease. 
However, it must be recognized that the success of a cervical cancer screening program won't be guaranteed by any method of screening, but by the overall organization of the system, providing high population coverage, fast access to testing results and easy admission to centers for treatment and follow-up of precursor lesions. Computerized web-based database accessible to health care providers is instrumental in managing the screening program and evaluating the efficacy of new initiatives, as the one here reported, planned to be soon implemented in São Paulo as a pilot experience.

\section{Financial Support}

This study was supported by research funds granted to the INCT-HPV (www.hpv.org.br) by the Conselho Nacional de Pesquisas (CNPq, Grant \# 573799/2008-3) and Fundação de Amparo a Pesquisa do Estado de São Paulo (FAPESP, Grant \# 2008/57889-1). All commercial tests employed were purchased by the INCT-HPV. Companies didn't have any role in the study design, data evaluation or manuscript writing.

\section{Disclosures}

L. L. Villa is a Roche consultant/advisory board member for HPV DNA diagnostic tests.

\section{References}

[1] Franco, E.L., Rohan, T.E. and Villa, L.L. (1999) Epidemiologic Evidence and Human Papillomavirus Infection as a Necessary Cause of Cervical Cancer. Journal of the National Cancer Institute, 17, 506-511. http://dx.doi.org/10.1093/jnci/91.6.506

[2] Zur Hausen, H. (2002) Papillomaviruses and Cancer: From Basic Studies to Clinical Application. Nature Reviews Cancer, 2, 342-350. http://dx.doi.org/10.1038/nrc798

[3] Mayrand, M.H., Duarte-Franco, E., Rodrigues, I., Walter, S.D., Hanley, J., Ferenczy, A., Ratnam, S., Coutlée, F. and Franco, E.L. (2007) Canadian Cervical Cancer Screening Trial Study Group. Human Papillomavirus DNA versus Papanicolaou Screening Tests for Cervical Cancer. The New England Journal of Medicine, 357, 1579-1588. http://dx.doi.org/10.1056/NEJMoa071430

[4] Roth, W.K., Busch, M.P., Schuller, A., Ismay, S., Cheng, A., Seed, C.R., et al. (2012) International Survey on NAT Testing of Blood Donations: Expanding Implementation and Yield from 1999 to 2009. Vox Sanguinis, 102, 82-90. http://dx.doi.org/10.1111/j.1423-0410.2011.01506.x

[5] Saslow, D., Solomon, D., Lawson, H.W., et al. (2012) American Cancer Society, American Society for Colposcopyand Cervical Pathology, and American Society for Clinical Pathology screening guidelines for the Prevention and Early Detection of Cervical Cancer. American Journal of Clinical Pathology, 137, 516-542. http://dx.doi.org/10.1309/AJCPTGD94EVRSJCG

[6] Naucler, P., Ryd, W., Tornberg, S., et al. (2007) Human Papillomavirus and Papanicolaou Tests to Screen for Cervical Cancer. The New England Journal of Medicine, 357, 1589-1597. http://dx.doi.org/10.1056/NEJMoa073204

[7] Bulkmans, N., Berkhof, J., Rozendaal, L., et al. (2007) Human Papillomavirus DNA Testing for the Detection of Cervical Intraepithelial Neoplasia Grade 3 and Cancer: 5-Year Follow-Up of a Randomised Controlled Implementation Trial. Lancet, 370, 796-802. http://dx.doi.org/10.1016/S0140-6736(07)61450-0

[8] Ronco, G., Giorgi-Rossi, P., Carozzi, F., Confortini, M., Dalla Palma, P., Del Mistro, A., et al. (2010) Efficacy of Human Papillomavirus Testing for the Detection of Invasive Cervical Cancers and Cervical Intraepithelial Neoplasia: A Randomised Controlled Trial. The Lancet Oncology, 11, 249-257. http://dx.doi.org/10.1016/S1470-2045(09)70360-2

[9] Schiffman, M., Wentzensen, N., Wacholder, S., Kinney, W., Gage, J.C. and Castle, P.E. (2011) Human Papillomavirus Testing in the Prevention of Cervical Cancer. Journal of the National Cancer Institute, 103, 368-383. http://dx.doi.org/10.1093/jnci/djq562

[10] Mühlberger, N., Sroczynski, G., Esteban, E., Mittendorf, T., Miksad, R.A. and Siebert, U. (2008) Cost-Effectiveness of Primarily Human Papillomavirus-Based Cervical Cancer Screening in Settings with Currently Established Pap Screening: A Systematic Review Commissioned by the German Federal Ministry of Health. The International Journal of Technology Assessment in Health Care, 24, 184-192. http://dx.doi.org/10.1017/S0266462308080264

[11] Levin, C.E., Sellors, J., Shi, J.F., Ma, L., Qiao, Y.L., Ortendahl, J., et al. (2010) Cost-Effectiveness Analysis of Cervical Cancer Prevention Based on a Rapid Human Papillomavirus Screening Test in a High-Risk Region of China. International Journal of Cancer, 127, 1404-1411. http://dx.doi.org/10.1002/ijc.25150

[12] Vijayaraghavan, A., Efrusy, M., Lindeque, G., Dreyer, G. and Santas, C. (2009) Cost Effectiveness of High-Risk HPV DNA Testing for Cervical Cancer Screening in South Africa. Gynecologic Oncology, 112, 377-383.

http://dx.doi.org/10.1016/j.ygyno.2008.08.030 
[13] Flores, Y.N., Bishai, D.M., Lorincz, A., Shah, K.V., Lazcano-Ponce, E., Hernández, M., Granados-García, V., Pérez, R. and Salmerón, J. (2011) HPV Testing for Cervical Cancer Screening Appears More Cost-Effective than Papanicolaou cytology in Mexico. Cancer Causes Control, 22, 261-272. http://dx.doi.org/10.1007/s10552-010-9694-3

[14] Berkhof, J., Coupé, V.M., Bogaards, J.A., van Kemenade, F.J., Helmerhorst, T.J., Snijders, P.J. and Meijer, C.J. (2010) The Health and Economic Effects of HPV DNA Screening in The Netherlands. International Journal of Cancer, 127, 2147-2158. http://dx.doi.org/10.1002/ijc.25211

[15] Rijkaart, D.C., Berkhof, J., van Kemenade, F.J., Coupe, V.M., Hesselink, A.T., Rozendaal, L., et al. (2012) Evaluation of 14 Triage Strategies for HPV DNA-Positive Women in Population-Based Cervical Screening. International Journal of Cancer, 130, 602-610. http://dx.doi.org/10.1002/ijc.26056

[16] Rijkaart, D.C., Berkhof, J., Rozendaal, L., van Kemenade, F.J., Bulkmans, N.W., Heideman, D.A., et al. (2012) Human Papillomavirus Testing for the Detection of High-Grade Cervical Intraepithelial Neoplasia and Cancer: Final Results of the POBASCAM Randomised Controlled Trial. The Lancet Oncology, 13, 78-88. http://dx.doi.org/10.1016/S1470-2045(11)70296-0

[17] Lazcano-Ponce, E. and Allen-Leigh, B. (2009) Innovation in Cervical Cancer Prevention and Control in Mexico. Archives of Medical Research, 40, 486-492. http://dx.doi.org/10.1016/j.arcmed.2009.07.007

[18] Silva, G.A., Gamarra, C.J., Girianelli, V.R. and Valente, J.G. (2011) Cancer Mortality Trends in Brazilian State Capitals and Other Municipalities between 1980 and 2006. Revista de Saúde Pública, 45, 1009-1018. http://dx.doi.org/10.1590/S0034-89102011005000076

[19] Cancer Incidence in Brazil (2011) Estimate for Year 2012/ Instituto Nacional de Câncer José Alencar Gomes da Silva, Coordenação Geral de Ações Estratégicas, Coordenação de Prevenção e Vigilância, Rio de Janeiro. http://www.inca.gov.br

[20] Gravitt, P.E., Belinson, J.L., Salmeron, J. and Shah, K.V. (2011) Looking Ahead: A Case for Human Papillomavirus Testing of Self-Sampled Vaginal Specimens as a Cervical Cancer Screening Strategy. International Journal of Cancer, 129, 517-527. http://dx.doi.org/10.1002/ijc.25974

[21] Fregnani, J.H., Scapulatempo, C., Haikel, Jr., R.L., Saccheto, T., Campacci, N., Mauad, E.C., Longatto-Filho, A., RODEO Study Team (2013) Could Alarmingly High Rates of Negative Diagnoses in Remote Rural Areas Be Minimized with Liquid-Based Cytology? Preliminary Results from the RODEO Study Team. Acta Cytologica, 57, 69-74. http://dx.doi.org/10.1159/000343046

[22] Newcombe, R.G. (1998) Two-Sided Confidence Intervals for the Single Proportion: Comparison of Seven Methods. Statistics in Medicine, 17, 857-872. http://dx.doi.org/10.1002/(SICI)1097-0258(19980430)17:8<857::AID-SIM777>3.0.CO;2-E

[23] Villa, L.L. (2008) Assessment of New Technologies for Cervical Cancer Screening. The Lancet Oncology, 9, $910-911$. http://dx.doi.org/10.1016/S1470-2045(08)70238-9

[24] Arbyn, M., Ronco, G., Meijer, C.J. and Naucler, P. (2009) Trials Comparing Cytology with Human Papillomavirus Screening. The Lancet Oncology, 10, 935-936. http://dx.doi.org/10.1016/S1470-2045(09)70296-7

[25] Leinonen, M., Nieminen, P., Kotaniemi-Talonen, L., Malila, N., Tarkkanen, J., Laurila, P. and Anttila, A (2009) AgeSpecific Evaluation of Primary Human Papillomavirus Screening vs Conventional Cytology in a Randomized Setting. Journal of the National Cancer Institute, 101, 1612-1623. http://dx.doi.org/10.1093/jnci/djp367

[26] Heideman, D.A., Hesselink, A.T., Berkhof, J., van Kemenade, F., Melchers, W.J., Daalmeijer, N.F., et al. (2011) Clinical Validation of the Cobas 4800 HPV Test for Cervical Screening Purposes. Journal of Clinical Microbiolog, 49, 3983-3985. http://dx.doi.org/10.1128/JCM.05552-11

[27] Meijer, C.J., Berkhof, J., Castle, P.E., Hesselink, A.T., Franco, E.L., Ronco, G., et al. (2009) Guidelines for Human Papillomavirus DNA Test Requirements for Primary Cervical Cancer Screening in Women 30 Years and Older. International Journal of Cancer, 124, 516-520. http://dx.doi.org/10.1002/ijc.24010

[28] Ayres, A.R. and Silva, G.A. (2010) Cervical HPV Infection in Brazil: Systematic Review. Revista de Saúde Pública, 44, 963-974.

[29] Schlecht, N.F., Platt, R.W., Duarte-Franco, E., Costa, M.C., Sobrinho, J.P., Prado, J.C., et al. (2003) Human Papillomavirus Infection and Time to Progression and Regression of Cervical Intraepithelial Neoplasia. Journal of the National Cancer Institute, 95, 1336-1343. http://dx.doi.org/10.1093/jnci/djg037

[30] Schopp, B., Holz, B., Zago, M., Stubenrauch, F., Petry, K.U., Kjaer, S.K. and Iftner, T. (2010) Evaluation of the Performance of the Novel Papillo Check HPV Genotyping Test by Comparison with Two Other Genotyping Systems and the HC2 Test. Journal of Medical Virology, 82, 605-615. http://dx.doi.org/10.1002/jmv.21719

[31] Wright, Jr., T.C., Stoler, M.H., Sharma, A., Zhang, G., Behrens, C., Wright, T.L., ATHENA (Addressing the Need for Advanced HPV Diagnostics) Study Group (2011) Evaluation of HPV-16 and HPV-18 Genotyping for the Triage of Women with High-Risk HPV+ Cytology-Negative Results. American Journal of Clinical Pathology, 136, 578-586. http://dx.doi.org/10.1309/AJCPTUS5EXAS6DKZ 